\title{
EXTRACTION OF WAVELET BASED FEATURES FOR CLASSIFICATION OF T2-WEIGHTED MRI BRAIN IMAGES
}

\author{
Ms. Yogita K.Dubey ${ }^{1}$ and Milind M.Mushrif ${ }^{2}$ \\ Department of Electronics and Telecommunication, YCCE, Nagpur, INDIA \\ ${ }^{1}$ yogeetakdubey@yahoo.co.in, ${ }^{2}$ milindmushrif@yahoo.com
}

\begin{abstract}
Extraction of discriminate features is very important task in classification algorithms. This paper presents technique for extraction cosine modulated feature for classification of the T2-weighted MRI images of human brain. Better discrimination and low design implementation complexity of the cosine-modulated wavelets has been effectively utilized to give better features and more accurate classification results. The proposed technique consists of two stages, namely, feature extraction, and classification. In the first stage, the energy features from MRI images are obtained from sub-band images obtained after decomposition using cosine modulated wavelet transform. In the classification stage, Mahalanobis distance metric is used to classify the image as normal or abnormal. Average Classification accuracy with a success rate of $100 \%$ has been obtained.
\end{abstract}

\section{KEYWORDS}

Brain MRI, Feature extraction, Classification, Cosine-modulated Wavelets

\section{INTRODUCTION}

Brain MRI is commonly used modality for detecting and visualizing brain tumors. It provides detailed information regarding healthy tissue (normal) and pathological process (abnormality). Clinical MRI is based on the hydrogen nucleus. For image formation, a large static magnetic field is used to perturb magnetic moments of proton that exist in the hydrogen nucleus from their equilibrium. Because of this, the protons are oriented randomly. But in existence of a static magnetic field, they line up with the field and the net magnetization of protons tends toward the direction of the field. In the relaxation process an induced electronic signal is recorded. The strength and duration of the signal depend on three quantities:

1. $\rho$ (proton density),

2. Spin-lattice relaxation time: the time which describes how fast the net magnetization takes to relax back to its equilibrium (T1),

3. Spin-spin relaxation time: with this time, magnetization components decrease to zero (T2).

In scanning of a person's body, by using different parameters setting, it is possible to obtain three different images of the same body: T1-weighted, T2-weighted, and $\rho$-weighted [1]. 
Classification of MR brain images plays a very important role for further processing of MR brain image which is helpful for the diagnosis of diseases. Many techniques for the classification of MR brain images are found in the literature. El-Syed et al [2] proposed a hybrid technique for brain MR images classification as tumorous and normal. In this technique discrete wavelet transform coefficients are used as attributes for classification. These attributes are reduced using Principal Component Analysis (PCA) before providing to the classifier. KNN and Feed forward back propagation neural network are used as classifier. They have reported a classification accuracy of $98.6 \%$ and $95.7 \%$. Ahmed Kharat et al [3] used genetic algorithm and support vector machine to classify the brain tissues into normal, benign or malignant tumor. The performance of the algorithm is evaluated on a series of brain tumor images. Qurat-ul-ain et al [4] proposed a method for classification and segmentation of brain tumor using texture feature in which texture features are extracted from brain MR image, then Brain images are classified on the bases of these texture feature using ensemble base classifier. Textures provide essential information and cues for the analysis of many image processing problems. One of the major applications of texture analysis is classification, which plays an important role in many applications such as tissue classification in medical imaging. Hsin [5] used a modulated wavelet transform approach for texture segmentation and reported that texture segmentation performance can be improved with this approach. Cosine-modulated wavelet based texture features are used by M. Kokare et al [6] for content-based image retrieval with good retrieval efficiency and accuracy. M. M. Mushrif et al [7] used cosine modulated wavelet transform based features for texture classification and reported improved classification rates compared to the traditional Gabor wavelet based approach, rotated wavelet filters based approach, DT-CWT approach and the DLBP approach.

In this paper, a technique extraction of cosine-modulated wavelets feature for the classification MRI brain images is proposed. The cosine modulated wavelets, introduced by Gopinath et al [8], have the advantages that they occupy adjacent bands in the spectrum that gives better frequency resolution and the analysis/synthesis computations in these wavelet bases can be done very efficiently using combination of two channel unitary filter banks and the discrete cosine transform. The computations of these wavelets have low design and implementation complexity, good filter quality, which yields improved performance in terms of classification accuracy and time.

The paper is organized as follows. Section 2 contains details of cosine modulated wavelet transform, Extraction of features from the sub-bands generated after cosine-modulated wavelet transform is explained in section 3. Detail description of the classification with training and testing algorithms is described in Section 4. Section 5 contains the results. Finally, conclusions are presented in Section 6.

\section{Cosine Modulated Wavelet Transform}

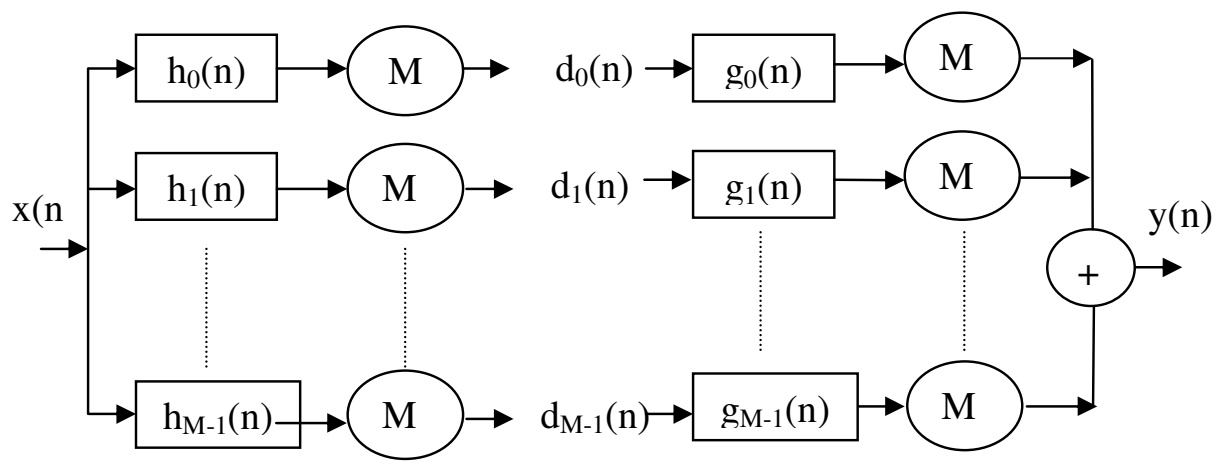

Figure 1. M Channel Filter bank 
Figure 1 shows an M-channel filter bank with analysis filters $h_{i}$ and synthesis filters $g_{i}$. Filter bank is said to be perfect reconstruction if $y(n)=x(n)$. A perfect reconstruction filter bank is unitary if $g_{i}(n)=h_{i}(-n)$. Koilpillai et al [9] reported that unitary (FIR) filter banks are practically important since they can be completely parameterized and efficiently implemented. Moreover, they give rise to orthonormal wavelet bases for $L^{2}(R)$. A unitary filter bank where the low-pass filter satisfies the additional linear constraints given in Eq. (1) gives rise to wavelet tight frames.

$$
\sum_{k=0}^{N-1} h_{0}(k)=\sqrt{M} \text {. }
$$

where $h_{0}(k)$ is lowpass filter of length $\mathrm{N}$, and the number of channels are $\mathrm{M}$. This filter is the unitary scaling vector, and the remaining filters in the filter bank are the unitary wavelet vectors. The scaling and wavelet vectors determine the scaling function, $\psi_{0}(t)$, and the $(M-1)$ wavelets, $\psi_{i}(t)$, are defined by

$$
\psi_{i}(t)=\sqrt{M} \sum_{k} h_{i}(k) \psi_{0}(M t-k) \quad i \in\{0,1, \ldots . M-1\}
$$

The $(M-1)$ wavelets $\psi_{i}(t) i \in\{0,1, \ldots M-1\}$ their translates and dilates by powers of $\mathrm{M}$ form a wavelet tight frame for $L^{2}(R)$ as reported by Gopinath et al [8].

For every function $f(t)=L^{2}(R)$, one has

$$
f(t)=\sum_{i=1}^{M-1} \sum_{j, k}\left\langle f, \psi_{i, j, k}(t)\right\rangle \psi_{i . j . k}(t)
$$

Where $\langle\cdot\rangle$ is an inner product and $\psi_{i, j, k}=M^{j / 2} \psi_{i}\left(M^{j} t-k\right)$.

Modulated filter banks are special class of filter banks where the analysis and synthesis filters are obtained by modulation of prototype filters. Koilpillai et al [9] had reported that Cosinemodulated FIR filter banks are a special class of unitary FIR filter banks, where analysis filters are all cosine modulates of a low-pass linear phase prototype filter. In an $M$-channel filter bank, the analysis and the synthesis filters are meant to approximate ideal $L^{\text {th }}$ band filters, the passbands of which occupy adjacent frequency channels that are $\pi / \mathrm{L}$ apart. If we modulate a real, prototype filter, with pass-band in $\left[-\frac{\pi}{2 M}, \frac{\pi}{2 M}\right]$ by $\cos \left((2 i+1) \frac{\pi}{2 M} n+\varepsilon_{i}\right)$, where $\varepsilon_{i}$ is an arbitrary phase, the pass-band of the resultant filter occupies the desired bands.. Here we used filter coefficients for $M=2$ to decompose the texture image in to four channels. The prototype filter $h_{0}$ and the analysis filter $h_{1}$ are related by

$$
h_{i}(n)=\cos \left(\frac{3 \pi}{4}\left(n-\frac{N-1}{2}-\frac{\pi}{4}\right)\right) h_{0}(n) \text {. }
$$

where $h_{0}(n)$ is an even-symmetric prototype filter of length $N=4 m$ for some nonnegative integer $\mathrm{m}$. After obtaining the prototype filter $h_{0}$ and the cosine modulated high-pass filter $h_{1}$, the wavelet 
decomposition is performed as explained in Figure 2 Each sub-band image contains the information of image at specific scale and orientation.

\section{Column}

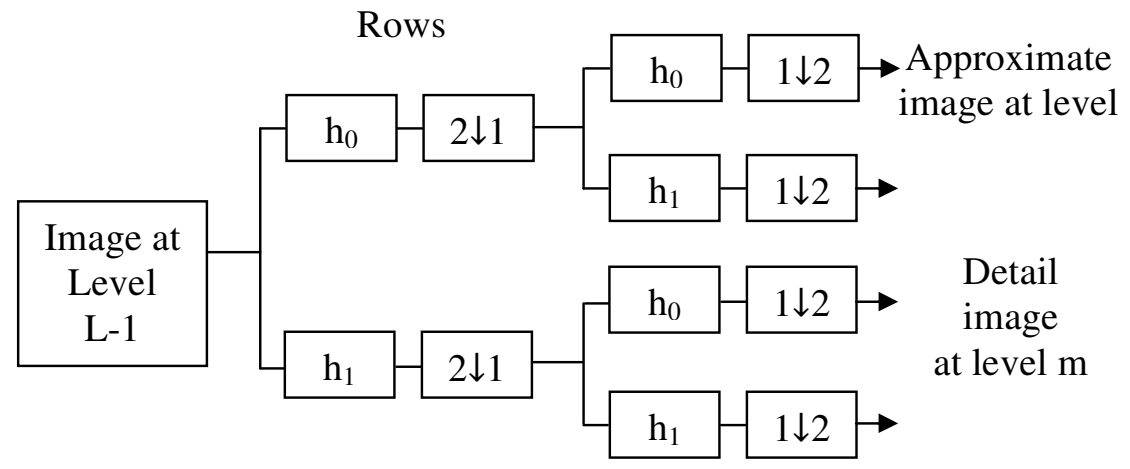

\section{$2 \downarrow 1$}

Keep one column out of two.

$1 \downarrow 2 \quad$ Keep one column out of two.

Figure 2. Filter bank used to calculate wavelet coefficients

\section{Feature eXtraction}

By applying the cosine-modulated wavelet transform, a number of sub-bands are generated. The magnitudes of wavelet coefficients in a particular sub-band are greater for images with a strong textural content at the frequency and orientation represented by that sub-band. Therefore, the texture of an image can be represented by a feature vector that contains the average coefficient magnitude, known as averaged energy function. The energy distribution has important discriminatory properties for images and as such can be used as a feature for texture classification. We used energy signature for extraction of texture features as it reflects the distribution of energy along the frequency axis over scale and orientation. The discriminatory properties of the energy distribution in sub-bands result in texture features that have been observed to yield good characterization of textures. The energy feature $e_{s}$ of the image is given by

$$
e_{s}=\frac{1}{M \times N} \sum_{i=1}^{M} \sum_{j=1}^{N}|x(i, j)| \text { for } \mathrm{s}=1,2, \ldots, Q
$$

where $x$ is wavelet decomposed image for any sub-band of dimension $M \times N$. For $K$-level decomposition of the image, the size of the feature vector is $Q=\left(3^{*} K+1\right)$. In the proposed work we used 2-level decomposition, so the resulting feature vector is of length 7 , given by $f=\left\{e_{1}, e_{2}, \ldots, e_{7}\right\}$.since different features have different range of possible values and the entire feature may not have the same the same level of significance because after decomposition of image, the sub-bands with higher resolution corresponds to noise and may not valuable for classification. So all these feature values are normalized in the range of 0 and 1 by the maximum value in the feature space before classification. 


\section{Classification Algorithm}

There are two image classes normal and abnormal. We have used Total 20 Brain MRI images for each class. For each image we calculate 7 features by calculating energy feature of each channel of the wavelet decomposition using equation (5). The classification algorithm is a two stage algorithm that involves training and classification (or testing) stages. The block diagram of algorithms is as follows

\section{Training Phase}

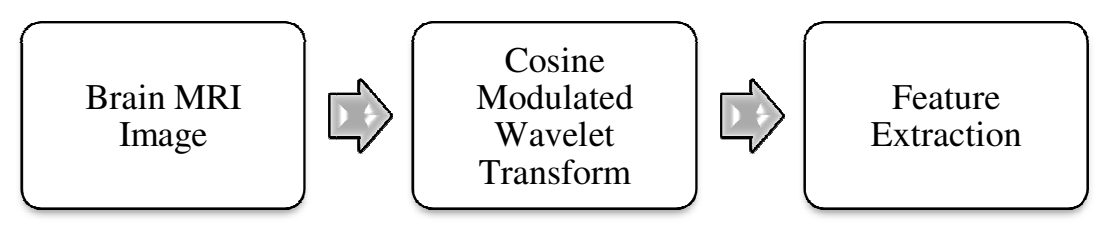

Figure 3(a). Block diagram of the proposed algorithm for training phase

\section{Classification Phase}

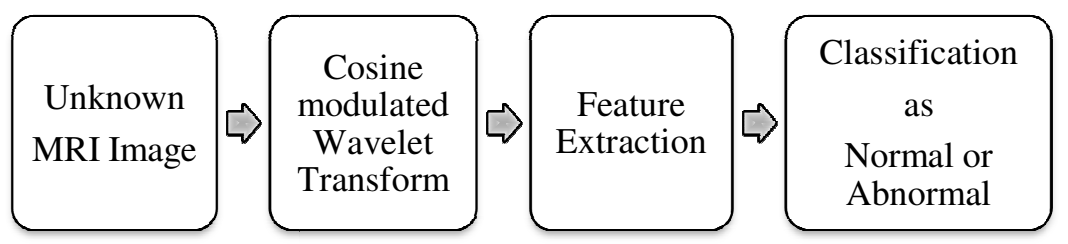

Figure 3(b). Block diagram of the proposed algorithm for classification phase

The algorithm is detailed as follow:

\subsection{Training Phase}

1. Decompose image I, using cosine-modulated wavelet transform.

2. Compute the energy feature of each channel of the wavelet decomposition using equation (5) and obtain a feature vector having $Q$ elements.

3. Repeat steps (1) and (2) for all training images of each class and calculate the cluster center vector $f m$ by taking average over all $N$ samples.

\subsection{Classification Phase}

1. Decompose an unknown brain MR image with the cosine-modulated wavelet transform. Compute the energy feature of each channel of the wavelet decomposition and obtain a feature vector $f$.

2. Calculate the decision function $D_{w}=D\left(f, f_{m}\right)$ for the unknown image using equation (6) given below 
Signal \& Image Processing : An International Journal (SIPIJ) Vol.3, No.1, February 2012

$$
d(f, f m)=(f-f m) C_{V}^{-1}(f-f m)^{T}
$$

where $\mathrm{fm}$ is the cluster center of feature vector. $C_{V}$ is the covariance of the feature vector.

3. Assign unknown image to a class $w$ if $D_{w} \leq D_{j}$ for all $j \neq w$ and $w=1,2$ (normal or abnormal).

\section{EXPERIMENTAL RESULTS}

The proposed technique is implemented on a real human brain images. The database consists of total 40 images: 20 normal and 20 abnormal images. These are $1.5 \mathrm{~T}$, axial, T2-weighted images of 300x400 sizes. These images are belonging to 18 persons ( 12 men and 06 women). Their ages vary between 7 and 80 years. The samples of normal and abnormal images are shown in Figure 4 .

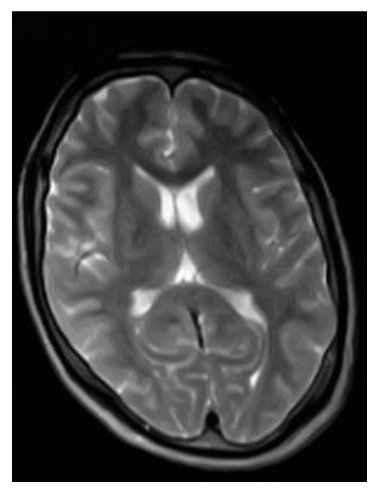

(a) Normal

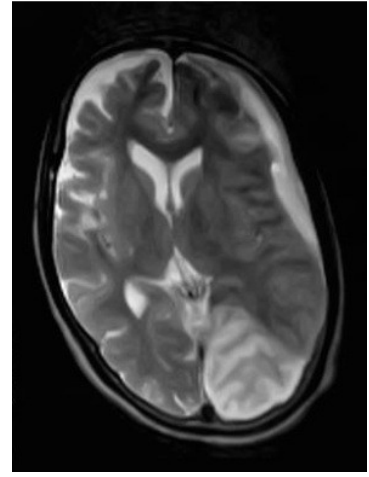

(b) Abnormal

Figure 4. Samples of normal and abnormal images in Axial view (T2 Weighted)

In figure 4(b), there is altered signal on left fronto- temporo-parietal region intensity appearing heterogeneously hyper intense indicates the possibility of lesion in that image. Out of 40 images, 7 images from each class is randomly selected for training the classifier and the remaining 13 from each class is used for testing the classification accuracy of the proposed technique. Thus there are $7 \times 2=14$ images for training and $13 \times 2=26$ images for classification.

The Proposed algorithm is applied to each image of the database. Two levels of wavelet decomposition with Cosine modulated wavelet and Daubechies wavelet of length 4 (db4) is done. After wavelet decomposition 7 sub-bands are generated, for each sub-band energy feature is calculated. Feature vector for all the 14 images of the database is created for training. For similarity matching in classification phase Bays classifier is used. For the query image, classification algorithm computes the feature vector and compares with all features of the feature vector. The similarity between query image and image in the database is calculated by Mahalanobis distance and query image labeled to its proper class (normal or abnormal) on the basis of minimum distance to the cluster center vector of that class. Cluster vector obtained alter two level of wavelet decomposition for Cosine modulated wavelet and Daubechies wavelet of length $4(\mathrm{db} 4)$ is shown in Table 1. 
Signal \& Image Processing : An International Journal (SIPIJ) Vol.3, No.1, February 2012

Table1. Cluster vector for normal and abnormal images of the database

\begin{tabular}{|c|c|c|c|c|c|c|c|c|}
\hline \multirow{2}{*}{ Wavelets } & \multirow{2}{*}{$\begin{array}{c}\text { Image } \\
\text { class }\end{array}$} & \multicolumn{6}{|c|}{ Energy feature of 7 sub bands obtained after 2 level wavelet } \\
\cline { 3 - 9 } & & LH1 & HL1 & HH1 & LL2 & LH2 & HL2 & HH2 \\
\hline \multirow{2}{*}{$\begin{array}{c}\text { Cosine } \\
\text { modulated } \\
\text { wavelet }\end{array}$} & NL & 0.0053 & 0.0079 & 0.0020 & 0.8234 & 0.0234 & 0.0378 & 0.0103 \\
\cline { 2 - 9 } & ANL & 0.0047 & 0.0064 & 0.0017 & 0.7783 & 0.0214 & 0.0321 & 0.0093 \\
\hline \multirow{2}{*}{$\begin{array}{c}\text { Daubechies } \\
\text { wavelet } \\
\text { (db4) }\end{array}$} & NL & 0.0043 & 0.0064 & 0.0043 & 0.8242 & 0.0198 & 0.0036 & 0.0096 \\
\cline { 2 - 9 } & ANL & 0.0039 & 0.0052 & 0.0039 & 0.7789 & 0.018 & 0.0308 & 0.0085 \\
\hline
\end{tabular}

NL: Normal brain MR image, ANL: Abnormal brain MR image.

LH1, HL1, HH1 and LH2, HL2, HH2 represent the finest scale wavelet coefficient i.e. detail image at level 1 and level 2 respectively and LL2 coarse level coefficients i.e. approximation image at level 2.

Experimental results with correct classification percentage are presented in Table 2. Very good average classification rate of $100 \%$ is achieved using only 7 energy features obtained from a 2 level decomposition of cosine-modulated wavelets against the accuracy of only $92.857 \%$ with wavelet transform using Daubechies wavelet (db4).

Table2. Percentage classification accuracy for normal and abnormal MR brain images

\begin{tabular}{|c|c|c|}
\hline & $\mathrm{db} 4$ wavelet & Cosine-modulated wavelets \\
\hline Normal image & 100 & 100 \\
\hline Abnormal image & 85.714 & 100 \\
\hline$\%$ Average Classification & 92.857 & 100 \\
\hline
\end{tabular}

The classification accuracy of this work shows the advantages of the proposed technique. Cosine modulated wavelet features gives better inter class variability as compared to the Daubechies wavelet features. Better discrimination and low design implementation complexity and good filter quality, of the cosine-modulated wavelets has been effectively utilized to give better features improved performance in terms of classification accuracy and time. 
Signal \& Image Processing : An International Journal (SIPIJ) Vol.3, No.1, February 2012

\section{CONCLUSiON}

In this paper, we have presented a technique for extraction of cosine-modulated wavelet feature for the classification of T2-weighted MR brain image. The two stage algorithm is applied on real brain images of 40 patients. Training is carried on $35 \%$ of image database and tested accurately on remaining $65 \%$ of image database. Mahalanobis distance metric is used to classify brain MRI image as normal or abnormal. The proposed classification algorithm gives very high classification accuracy of $100 \%$ with the available database of MR brain images. The work can be extended to develop the algorithms for localization of tumor in abnormal images as well as for differentiating and grading of pathological brain tissues (e.g. necrotic tissue, lesion, edema etc) in MR brain image which will be helpful to detect the early stage changes taking place in brain.

\section{ACKNOWLEDGEMENTS}

The authors would like to thank Dr. Kajal Mitra, Prof. \& Head, Radiolgy and Diagnostic Imaging department, NKP Salve Institute and Lata Mangeshkar Hospital, Nagpur, for providing brain MR images and for help in interpretation and validation of result.

\section{REFERENCES}

[1] M.A.Balfar,A.R.Ramli,M.I.Saripan,S. Mashohor, “ Review of Brain MRI image segmentation Method”, Artificial Intelligent Rev (2010) 33: 261-274

[2] El-Sayed A, El Dahshan, Abdel Badeem M, Salem, Tamer H. Younis, "A Hybrid technique for MRI brain image classification”. Studia University, Babes-Bolyai,Informatica, Volume LIV, November, 2009

[3] Ahmed Kharrat, Karim Gasmi,Mohamad Ben Messaoud, Nacera Benamarene, Mohamad Abid, "A Hybrid approach for automatic classification of Brain MRI using Genetic algorithm and support vector machine”, Leonardo journal of science, ISSN 1583-0233, Issue 17, July December 2010

[4] Qurat-ul-ain, Ghajanfar Latif, Sidra Batool Kajmi, M.Arphan Jafar, Anwar M. Mirja, Classification and segmentation of Brain tumor using texture features, Recent Advance in artificial intelligence, Knowledge engineering and databases.

[5] Hsin H.C. Texture segmentation using modulated wavelet transforms. IEEE Transactions on Image Processing. 2000. 9 (7), pp. 1299-1302.

[6] Manesh Kokre, B.N.Chatterji,P.K.Biswas, "Cosine Modulated wavelet based texture features for content- based image retrieval”, Pattern recognition letters (25) (2004) 391-398

[7] M.M.Mushrif, Yogita K.Dubey, "Texture Classification using Cosine modulated wavelets", 3rd International conference on Signal Acquisition and Processing (ICSAP 2011), Singapore.

[8] R. A. Gopinath and C. S. Burrus. On cosine-modulated wavelet orthonormal bases. IEEE Transactions on Image Processing, 4(2):162-176, 1995.

[9] R. D. Koilpillai and P. P. Vaidyanathan. Cosine-modulated filter banks satisfying perfect reconstruction. IEEE Transactions on Signal Processing, 40(4):770-783, 1992. 


\section{Authors}

Ms. Yogita K.Dubey received the B.E. in Electronics Engineering from MPIET, Gondia and MTech in Electronics from YCCE, Nagpur. She is currently pursuing $\mathrm{PhD}$ from RTM Nagpur University and working as a Asst. Prof. in Department of Electronics and Telec ommunication, Yeshwantrao Chavan College of Engineering, Nagpur, India since 2003. Her research interests include image processing, computer vision and medical imaging.

Dr. Milind M. Mushrif received the B.E. in Electrical Engineering and the M.E. in Electronics Engineering from Walchand College of Engineering, Sangli and Ph.D. degree from IIT Kharagpur. He joined Yeshwantrao Chavan College of Engineering in 1990, where he is currently a Professor in Electronics and Telecommunication Engineering. He has more than 30 research publications in national and international journals and conferences. His main interests are in computer vision, pattern recognition and soft computing techniques.
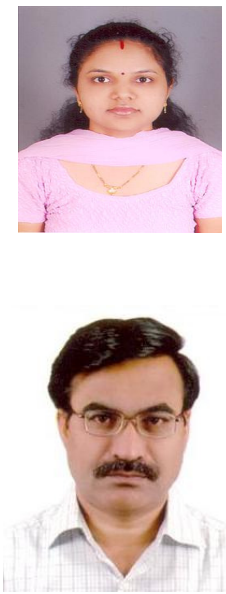